\title{
An Introduction to Light-emitting Diodes
}

\author{
C. Michael Bourget \\ Orbital Technologies Corporation, Electrical Engineering, 1212 Fourier Drive, Madison, WI 53717
}

\author{
Additional index words. controlled environment, lighting, efficiency, fluorescent, HID, light source
}

\begin{abstract}
Light-emitting diodes (LEDs) are semiconductor devices that produce noncoherent, narrow-spectrum light when forward voltage is applied. LEDs range in wavelength from the UVC band to infrared (IR) and are available in packages ranging from milliwatts to more than $10 \mathrm{~W}$. The first LED was an IR-emitting device and was patented in 1961. In 1962, the first practical visible spectrum LED was developed. The first high-power (1-W) LEDs were developed in the late 1990s. LEDs create light through a semiconductor process rather than with a superheated element, ionized gas, or an arc discharge as in traditional light sources. The wavelength of the light emitted is determined by the materials used to form the semiconductor junction. LEDs produce more light per electrical watt than incandescent lamps with the latest devices rivaling fluorescent tubes in energy efficiency. They are solid-state devices, which are much more robust than any glassenvelope lamp and contain no hazardous materials like fluorescent lamps. LEDs also have a much longer lifetime than incandescent, fluorescent, and high-density discharge lamps (U.S. Dept. of Energy). Although LEDs possess many advantages over traditional light sources, a total system approach must be considered when designing an LED-based lighting system. LEDs do not radiate heat directly, but do produce heat that must be removed to ensure maximum performance and lifetime. LEDs require a constant-current DC power source rather than a standard AC line voltage. Finally, because LEDs are directional light sources, external optics may be necessary to produce the desired light distribution. A properly designed LED light system is capable of providing performance and a lifetime well beyond any traditional lighting source.
\end{abstract}

In the area of controlled environment agriculture (CEA), one of the most commonly discussed topics is that of lighting. The lighting system is generally the most expensive component of a CEA facility in terms of upfront costs as well as ongoing expenses (electricity use as well as replacement lamps). Naturally, advances in lighting technology are of great interest to the CEA community for these very reasons. Lightemitting diodes (LEDs) are the most recent lighting technology to enter the CEA arena and have great potential to improve performance and reduce the overall cost of controlled environment lighting.

\section{THE BASICS}

An LED is a solid-state semiconductor device. That is, it is more closely related to a computer chip than a light bulb. More specifically, it is a semiconductor device with a single junction. By intentionally introducing impurities (a process called "doping") into the semiconductor's chemical structure, its electrical properties can be changed. By doping the semiconductor with different materials at different locations, a junction can be formed between two areas of differently doped material.

Like a regular diode, electrical current flows very easily across the junction in one direction, but not the other. When current is flowing, the LED is said to be forwardbiased. LEDs only emit light when they are forward-biased. The color of the light emitted is determined by the type of semiconductor material and the impurities used to form the junction. LEDs can have peak emission wavelengths from $250 \mathrm{~nm}$ (UVC) to 1000 nm (infrared) and up.

Received for publication 18 Mar. 2008. Accepted for publication 2 Apr. 2008.
An LED is not a laser (although laser diodes do exist, they are a completely different device). LED light is of a noncoherent, narrow band (Wikipedia LED, 2008). In general, LEDs are not dangerous. However, care should be taken if LEDs in the UVB and UVC range are used, because those wavelengths can be dangerous to the eyes and skin in sufficient power levels. Also, some LEDs are very bright and should not be looked at directly for the same reason the sun should not be.

Single LEDs are commercially available in packages that require a few milliwatts up to $5 \mathrm{~W}$ or more. Prepackaged arrays that contain many LEDs and require $100+\mathrm{W}$ are also offered. The de facto standard for a modern, high-power LED is a $1-\mathrm{W}$ device with a nominal forward current of $350 \mathrm{~mA}$ at $3 \mathrm{~V}$. However, single LEDs can be capable of operating at currents as high as 1.5 amperes.

Although the LED was invented in the 1920 s, a practical visible-light version (red) was not developed until the early 1960s (Zheludev, 2007). These early devices were of very low power and were usable only as indicator lamps. Through the 1970s, developments continued, and shorter wavelengths (orange, yellow, and green) came onto the market. The first practical blue LED was developed in 1993 (Nakamura et al., 1996), and in 1996, a phosphor coating was applied to a blue LED to create the world's first white LED. The most recent major advance in LED technology was the development of the highpower (1 W or greater) device in 1999. This has led to a revolution across the lighting field, with agricultural lighting being no exception.

\section{ADVANTAGES}

So why have LEDs created such a stir in the lighting world? The two most commonly cited LED advantages are efficiency and lifetime. The newest high-power LEDs are more efficient (radiated power output divided by electrical power input) than incandescent and fluorescent lamps and are roughly equivalent to high-intensity discharge (HID) lamps (Fig. 1) (U.S. Dept. of Energy, 2005; Wikipedia Luminous Efficacy, 2008).

White LEDs are quite a bit less efficient than monochromatic versions because they rely on a phosphor coating to react with a monochromatic LED (usually blue) emission to create white light. Much of the efficiency loss occurs during this phosphor conversion (fluorescence) process.

The measurement of LED lifetime is somewhat subjective because LEDs do not generally "burn out" like traditional lamps; they tend to slowly become dimmer over time. The most commonly used "lifetime" metric for LEDs is to measure the time for the LED to $\operatorname{dim}$ to $70 \%$ of its original intensity when run at its nominal operating current and temperature (Philips Lumileds Lighting Company, 2008). Most high-power LEDs have a published lifetime specification of $\approx 50,000$ h (Fig. 2).

This is two to three times better than fluorescent or HID lamps and a 50-fold increase over typical incandescent lamps (U.S. Dept. of Energy, 2005).

LEDs are very rugged devices. There is no fragile glass envelop to break, and their lifetime is not shortened by repeated on/off cycles like with incandescent, fluorescent, and arc-discharge lamps. Fluorescent lamps contain mercury, which can cause problems if they are accidentally broken. Most municipalities also require special disposal of these lamps to properly handle the mercury, usually for an extra fee. LEDs contain no hazardous materials, so this is not an issue.

The small size of LEDs allows unprecedented flexibility in lighting fixture design. Rather than large, bulky fixtures to house bulbs, reflectors, and ballasts, LEDs may be housed in low-profile fixtures that are much more space-efficient. 


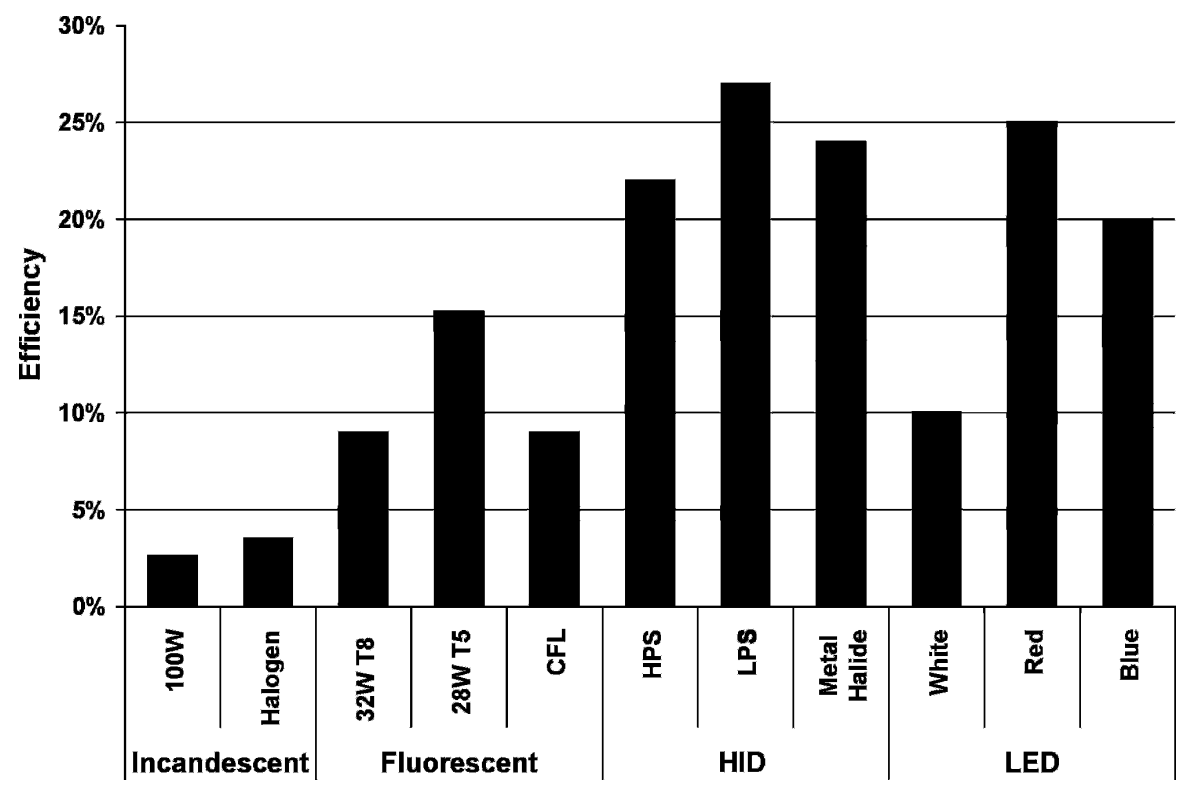

Fig. 1. A comparison of the luminous efficiencies (light energy output/electrical energy input) of selected electric light sources.

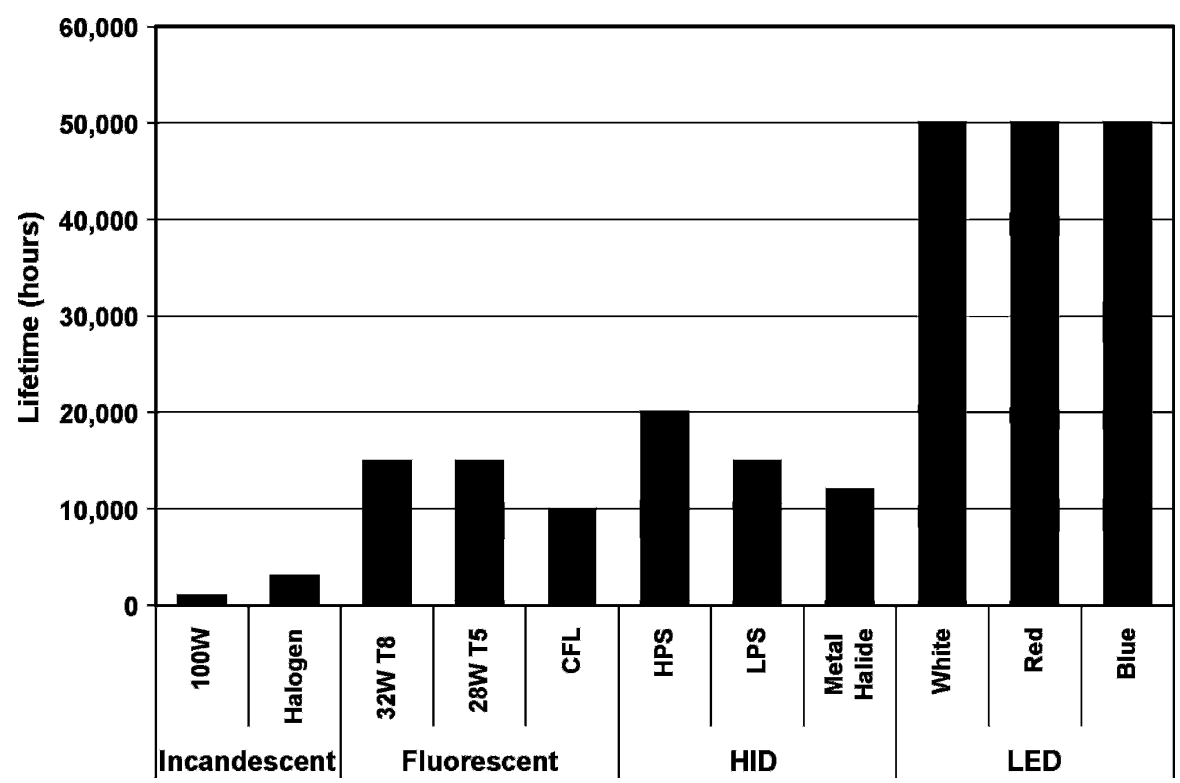

Fig. 2. A comparison of the lifetimes of selected electric lamps.

It is a common misconception that LEDs do not create any heat. LEDs do indeed create heat, but very little of that heat is actually radiated within the light beam. The vast majority of heat from the LED is conducted out of the rear of the device and into the mounting surface (heat sink). This allows LEDs to be placed very close to crops without the risk of a hot light beam scorching the plants. Consideration must be taken when designing the fixture to properly conduct the heat away from the LED, or the device's lifetime could be shortened considerably.

\section{APPLICATION AND SYSTEM DESIGN}

This very important point is the foundation of LED system design. Although LEDs possess many advantages over traditional lighting systems, they cannot simply be purchased, wired up, and turned on if a high performance level is expected. Careful thought must be placed into the design of the thermal rejection, power supply, control, and optical subsystems of any LED lighting system.

As discussed previously, the most important element of any LED system is the thermal design. Several very different LED cooling mechanisms are available, and each is appropriate for certain applications but not others. Direct conduction to a mounting surface, natural convection, forced-air (fancooled), and liquid cooling are all viable cooling systems for LEDs. The choice of the thermal system is determined by the size and application of the LED system. For example, a simple LED flashlight does not require a liquid cooling system; direct conduction to the outer case would be sufficient. However, in a large horticultural lighting system, a chilled water system may be appropriate to efficiently remove the heat.

LEDs are current controlled devices; they are designed to operate best when the amount of electrical current flowing through them is controlled. Because LEDs are diodes, they will tend to conduct a large amount of current (potentially much greater than their maximum safe limit) when forward-biased. This will happen if the LED is connected directly to a power source of a voltage greater than the LED's specified value and can potentially damage or destroy the LED. A circuit called a driver is used to regulate the current flowing through LEDs. There are many different driver designs and topologies available.

The driver is the interface between the LED and its power source. It regulates the current flowing through the LED to ensure that it is not operated beyond its limits. Drivers can be simple or complex depending on the application requirements. For example, if high efficiency and tight current regulation are not required, a single resistor can function as a simple current-limiting driver. On the other hand, complex switchmode drivers can be specifically designed to provide exact control and very high efficiency. Commercially available LED drivers suitable for small- to medium-sized applications are available. These are solely designed for driving LEDs and can greatly simplify system design. Some of these even include a builtin power supply and can be plugged directly into a standard wall outlet.

The power source must also be considered when designing large LED systems. For large LED lighting systems, industrial-type power supplies tend to be more appropriate. No matter what type of power source is selected, an appropriate driver must be used to ensure proper LED operation.

LED control systems allow the user to set various parameters of system operation, including brightness, time on or off (photoperiods), light quality, and color mixing (if multiple LED colors are used). Controls are usually interfaced to the driver circuitry to allow the brightness of the individual LEDs to be set. The control system can be as simple or complex as the application demands. Controls can be anything from manual adjustments to fully automated computer systems.

The final consideration when designing LED systems is that of optics. The proper optical design of an LED system can greatly reduce the number of LEDs required. This reduces hardware costs as well as electrical power use. Many types of standard lenses are available for the most popular styles of highpower LEDs. These are typically installed on each LED in the system. Reflectors like those used with HID lamps are generally ineffective because LEDs are directional sources. 


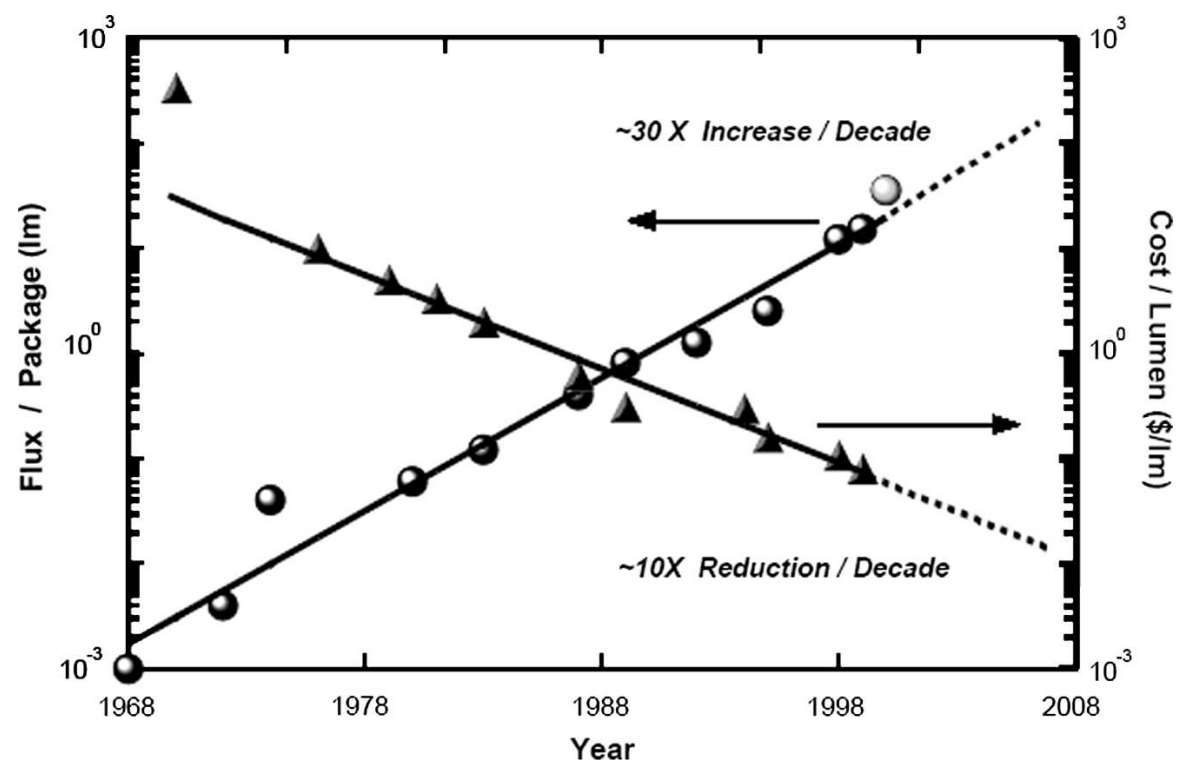

Fig. 3. A comparison of light-emitting diode costs and light output versus time.

Individual lenses are very effective in shaping and directing the light beam and can greatly increase light intensity at the desired location.

LEDs have found their way into almost every imaginable lighting application. From their first applications as simple indicator lamps and data displays, LEDs are now used in myriad applications. Some examples include scoreboards and large video boards ("jumbotrons"), traffic lights, street lights, vehicle lights (headlights, taillights, police lights, aircraft, and spacecraft), architectural accent lighting, and general lighting.
Of course, LED lighting also has a bright future in the world of horticultural lighting. Energy efficiency, long lifetime, and application flexibility make LEDs a natural choice for future agricultural lighting systems. Since the first practical LEDs were developed in the late $1960 \mathrm{~s}$, performance has been continuously increasing, whereas the cost per unit light output has been simultaneously dropping (Fig. 3) (Drennen et al., 2001). When applied in a well-designed system, no other light source can match the capabilities that LEDs have to offer.

\section{Recommended Reading}

Craford, M.G., N. Holonyak, and F.A. Kish. 2001. In pursuit of the ultimate lamp. Sci. Amer. 284:63-67.

Lighting Design Lab. 2008. Introduction to LED lighting. Mar. 2008. <http://lightingdesignlab. com/articles/LED_fund/intro_ledfund.htm>.

Philips Lumileds Lighting Company. 2008. Mar. 2008. <http://www.lumileds.com>.

Rensselaer Polytechnic Institute. 2008. Mar. 2008. $<$ http://www.lrc.rpi.edu/researchAreas/leds.asp $>$.

U.S. Dept. of Energy. 2008. Using LEDs for general illumination. Mar. 2008. <http://www.netl.doe. gov/ssl/usingLEDs/index.htm>.

Zukauskas, A., M.S. Shur, and R. Gaska. 2002. Introduction to solid state lighting. Wiley, New York, NY.

\section{Literature Cited}

Drennen, T., R. Haitz, and J. Tsao. 2001. A market diffusion and energy impact model for solidstate lighting. Sandia National Laboratories, SAND2001-2830J. August.

Nakamura et al. 1996. Light emitting gallium nitride-based compound semiconductor device. U.S. Patent 5,578,839, filed Nov. 17, 1993 and issued Nov. 26, 1996.

Philips Lumileds Lighting Company. 2008. Luxeon Rebel technical data sheet DS56. Mar. 2008. $<$ http://www.luxeon.com/pdfs/DS56.pdf $>$.

U.S. Dept. of Energy. 2005. Mar. 2008. <http://www. eere.energy.gov/consumer/your_home/lighting daylighting/index.cfm/mytopic $=12030>$.

Wikipedia. 2008. LED. Mar. 2008. <http://en. wikipedia.org/wiki/LED>.

Wikipedia. 2008. Luminous Efficacy. Mar. 2008. $<$ http://en.wikipedia.org/wiki/Luminous_efficacy $>$.

Zheludev, N. 2007. The life and times of the LED - A 100-year history. Nature Photonics 1:189-192. 